\title{
Generalidades sobre la migración de bagres amazónicos de la familia Pimelodidae y su relación con los ciclos hidrológicos
}

\author{
General information about the migration of Amazonian catfish from \\ the Pimelodidae family and its relationship with hydrological cycles
}

\section{Informações gerais sobre a migração dos bagres da Amazônia da família Pimelodidae e sua relação com os ciclos hidrológicos}

\author{
Jonathan F. Villamil-Rodríguez ${ }^{1 *}$; Leandro Cortés-Ávila ${ }^{2}$; José A. Rodríguez-Pulido ${ }^{*}$
}

1 Lic. en Prod. Agrop, Esp, MSc, Instituto de Acuicultura, Instituto de Acuicultura, Facultad de Ciencias Agropecuarias y Recursos Naturales, Universidad de los Llanos, Villavicencio, Colombia.

2 Biol, MSc, PhD, Profesor Colaborador, Programa de Tecnología en Producción Pesquera, Universidade do Estado do Amazonas, UEA - Manaos, Brasil.

3 Biol, MSc, (C)PhD, Profesor Universidad de los Llanos, Facultad de Ciencias Básicas e Ingeniería, Villavicencio, Colombia.

* Grupo de Investigación sobre Reproducción y Toxicología de Organismos Acuáticos - GRITOX. Instituto de Acuicultura, Universidad de los Llanos, Villavicencio, Colombia

Email: jonathan01.Ipa@gmail.com

\begin{abstract}
Resumen
El entendimiento de los eventos migratorios en peces dulceacuícolas es fundamental para conocer su biología y ecología, pues estos acontecimientos naturales conllevan a generar alternativas para la gestión y preservación de las pesquerías, especialmente en especies que presentan desafíos a causa de su gran capacidad de desplazamiento en toda la cuenca amazónica. Los grandes bagres de la familia Pimelodidae son caracterizados por presentar estas características migratorias, las cuales están influenciadas por el pulso de inundación, donde el incremento de las aguas por grandes precipitaciones en las cabeceras elevan el nivel de las aguas, estimulándolos a iniciar migraciones y a llevar a cabo el proceso de maduración gonadal, induciendo a los individuos a dirigirse a las áreas de desove río arriba para culminar su proceso reproductivo. El objetivo de la presente revisión, pretende abordar de forma general aspectos involucrados en la migración de peces Siluriformes y su relación con los ciclos hidrológicos.
\end{abstract}

Palabras clave: Planicie de inundación, potamodromo, Brachyplatystoma, estuario.

\begin{abstract}
The understanding of the migratory events in freshwater fish is fundamental to know their biology and ecology, since these natural events lead to generate alternatives for the management and preservation of fisheries, especially in species that present challenges due to their great capacity of displacement in the entire Amazon basin. The large catfish of the Pimelodidae family are characterized by presenting these migratory characteristics, which are influenced by the flood pulse, where the
\end{abstract}


increase of waters due to heavy rainfall in the headwaters raises the level of the waters, stimulating them to initiate migrations and to carry performed the process of gonadal maturation, inducing individuals to go to spawning areas upstream to complete their reproductive process. The objective of the present review, aims to address in a general way to deal with aspects involved in the migration of Siluriform fishes and their relationship with hydrological cycles.

Key words: Floodplain, potamodrome, Brachyplatystoma, estuary.

\section{Resumo}

O entendimento dos eventos migratórios em peixes de água doce é fundamental para conhecer os aspectos biológicos e ecológicos das espécies, este tipo de fenômenos naturais podem nos orientar a gerar alternativas para a gestão e preservação dos recursos pesqueiros, especialmente daquelas espécies que apresentam a capacidade de se deslocarem por toda a bacia amazônica. Os grandes bagres da família Pimelodidae são caracterizados por apresentar este tipo migração, onde ao estarem diretamente influenciados pelo pulso de inundação, especificamente quando ocorre aumento do nível das águas pelas precipitações na região andina, são estimulados a iniciarem migrações reprodutivas, induzindo-os a se dirigirem rio acima nas cabeceiras para culminar seu processo reprodutivo. O objetivo desta revisão foi abordar de forma geral os aspectos envolvidos na migração de peixes da ordem Siluriformes e sua relação com os períodos hidrométricos

Palavras chave: Planície de inundação, potamódromo, Brachyplatystoma, estuário.

\section{Introducción}

La biodiversidad es una de las palabras idóneas para definir a los ecosistemas acuáticos tropicales, debido a sus innumerables cuerpos hídricos que incluyen desde pequeños riachos a grandes ríos con diferentes tipos de agua y con diferentes características biológicas, químicas y físicas (Val et al., 2005). Un único cuerpo de agua puede ser susceptible a diversos cambios durante todo el año, incluso desapareciendo, en algunos casos, durante la estación seca (Junk et al., 1989; Almeida-Val et al., 1999) tal como ocurre en lagos de poca profundidad en el Amazonas (Sioli, 1984; Junk et al., 1989; Val y Almeida-Val, 1995). Geográficamente, la cuenca Amazónica cubre 7.05 millones de km², ocupando aproximadamente el 39\% de Sudamérica y alrededor del $72 \%$ se encuentra en Brasil (5.1 millones de $\mathrm{km}^{2}$ ) (Webb, 1995; Rossetti y Toledo, 1998). Posee una gran variedad de ambientes acuáticos, entre los cuales se destacan playas, riachuelos, cascadas y ríos de gran porte, bien sea de aguas claras, negras y blancas (Sioli, 1967; Lowe-McConnel, 1987).

Sus planicies de inundación y afluentes forman una red compleja de canales que a su vez se alteran conforme a la dinámica ambiental (Junk et al., 1989) promoviendo cambios limnológicos reflejados en los niveles de oxígeno, temperatura, disponibilidad alimenticia, refugio y áreas de cría para los peces (Junk et al., 1989; Nolan et al., 2009). Alteraciones en los parámetros ambientales y su interacción con factores endógenos en los peces, funcionan como un gatillo para eventos fisiológicos y comportamentales, desencadenando una serie de sucesos que intervienen en los procesos de migración, maduración de gametos y desove (LoweMcConnel, 1987; Nahagama et al., 1995, 2008).

De acuerdo al proceso migratorio, se generaliza que está presente para todo el reino animal, pero en peces, dicho acontecimiento permite aprovechar la variabilidad de nuevos hábitats y evitar condiciones adversas (Dingle y Drake, 2007; Binder et al., 2011). Según Mehner y Kasprzak (2011) el término migración, se refiere los movimientos definidos por individuos o poblaciones (o parte de poblaciones) entre dos hábitats, que hasta cierto punto, es predecible temporalmente y tiene una periodicidad regular. Por lo tanto, la migración difiere de la dispersión en que los individuos hacen un viaje de regreso al hábitat inicial. Este tipo de desplazamientos inician con base a una respuesta que tuvo la especie ante un estímulo ambiental y finalizan en cuanto dicho estímulo ya no está presente (Zapata y Usma, 2013).

Publicaciones referentes al estudio de la ecología de peces amazónicos como los realizados por Lowe-McConnel (1987) y Barthem y Fabré (2004) se han enfocado en proponer categorías y estrategias particulares de manejo de los peces de acuerdo a las características ambientales donde viven y el tipo de movimiento ejercido (longitudinal y lateral) en el ecosistema acuático, dando a conocer tres tipos: especies sedentarias, especies migratorias que hacen uso las planicies inundables y especies migratorias que llevan a cabo movimientos de grandes distancias. Los peces son el grupo taxonómico de mayor importancia en la región ama- 
zónica, pero sus patrones ecológicos no se conocen completamente (Granado-Lorencio et al., 2005; Pazin et al., 2006).

Numerosas especies son capturadas con fines ornamentales y de consumo en el ríos amazónicos (Bayley y Petrere Jr, 1989; Batista y Petrere Jr, 2003) sin embargo, órdenes como los Characiformes y Siluriformes se han establecido como los de mayor prevalencia en las pesquerías y en las investigaciones de carácter ecológico, donde los aportes científicos sobre composición de la estructura trófica en lagos y canales principales de la cuenca han sido de gran relevancia (Junk et al., 1983; Soares et al., 1986; Petry et al., 2003; CoxFernandes et al., 2004). La riqueza de los Siluriformes representa hasta el 39\% de las especies de la fauna Neotropical y la familia Pimelodidae es una de las más representativas, dentro de ella, algunas especies de los géneros Brachyplatystoma y Pseudoplatystoma son los que se destacan como migradores (Lowe-McConnel, 1987; Galvis et al., 1997; Barthem y Goulding, 1997).

Finalmente, aunque existe un interrogante cuando se intentan clasificar los diferentes tipos de migración en peces, algunos autores, hacen uso de ciertos términos para describirlos, entre estos: oceanódromos, potádromos y diádromos (Myers, 1949; McDowall, 1988). El objetivo de esta revisión ofrece algunas consideraciones generales sobre la migración de algunas especies de importancia en la familia Pimelodidae, nativos de la cuenca Amazónica, estrictamente considerados como potamodromos y su relación con los ciclos hidrológicos.

\section{Importancia de la actividad pesquera en la Amazonia de Colombia y Brasil}

La pesca hace parte intrínseca de la cultura ribereña, adaptándose a condiciones adversas en invierno y verano durante la subienda y bajanza de las aguas, pero en busca de satisfacer las necesidades de seguridad alimentaria, el pescador artesanal hace uso del producto de su trabajo para su subsistencia (Gualdrón, 2002; Diegues, 2004). En la Amazonia colombiana, esta labor es destacada por ser una de las actividades de impacto económico y cultural, que además de generar ingresos por la venta de pescado de consumo, también ha permitido incursionar en el mercado ornamental (Barthem et al., 1995; Fabré y Alonso, 1998; Agudelo et al., 2006).

Ante el anterior panorama, se desarrolla una recopilación de datos del comercio pesquero, cuya actividad es destacada en Leticia, Florencia, Puerto Leguízamo, Puerto Nariño y Mitú, pero es el municipio de Leticia que se ha caracterizado por ser uno de los principales centros de acopio de peces de cuero, teniendo también incidencia en parte del territorio peruano (Agudelo et al., 2006; De la Hoz y Manjarrés-Martínez, 2016). En los desembarcos de los Pimelodidae registrados por De la Hoz y Manjarrés-Martínez (2016) se encontraron especies como pintadillo tigre (Pseudoplatystoma tigrinum), dorado (Brachyplatystoma rousseauxii), amarillo (Zungaro zungaro), cajaro (Phractocephalus hemioliopterus), jugador (Brachyplatystoma juruense), pirabutón (Brachyplatystoma vaillantii), simi (Calophysus macropterus), barbiancho (Pinirampus pinirampus) y valentón (Brachyplatystoma filamentosum). Aun así, existe una problemática que se cierne en la Amazonia colombiana, como la carencia de lineamientos específicos que contribuyan al ordenamiento de la pesca artesanal (Agudelo, 2015) ya que tan sólo se han logrado estandarizar medidas enfocadas a las vedas, regulación de artes de pesca, áreas de pesca y tallas mínimas de captura, esto mismo acontece en lugares fronterizos de Perú y Brasil (Agudelo et al., 2009). Adicionalmente, una de las regulaciones implementadas entre Colombia y Brasil, fue la prohibición de la pesca y el comercio de C. macropterus, como consecuencia del uso indebido de carnadas de especies en peligro de extinción para su captura, como lo son los delfines Inia geoffrensis y Sotalia fluviatilis y los caimanes Melanosuchus niger y Caiman crocodilus, así, con esta medida se podría contribuir a la conservación de las especies, haciendo que C. macropterus fuera excluida dentro del mercado de bagres en la Amazonia brasilera (Iriarte y Marmontel, 2013; Brum et al., 2015).

Por otra parte, la pesca en la Amazonia de Brasil se encuentra desarrollada y posee intereses económicos, siendo el dorado (Brachyplatystoma rousseauxii) y el pirabutón (Brachyplatystoma vaillantii) los de mayor importancia y son las ciudades de Belén, Macapá, Santarém, Porto Velho, Lábrea, Tabatinga y Manaos, las zonas más sobresalientes (Batista et al., 2005; Barthem, 2013). En vista de esto, en Manaos y a través de ProVárzea se ha hecho el esfuerzo por evaluar el stock pesquero, permitiendo que últimamente sea un centro de acopio de importancia en la Amazonia (Almeida, 2006) debido a que la perspectiva de pesca pasó de ser artesanal a industrial. Sumado a esto, se han establecido áreas específicas para la delimitación de la actividad pesquera de grandes bagres, siendo desde Manaos (Brasil) hasta Iquitos (Perú) respectivamente (Barthem y Goulding, 1997). Aunque geográficamente, estos lugares han sido estratégicos para su comercialización, ecológicamente las distancias recorridas por los grandes bagres migradores indican que estas áreas están influenciadas por las cuatro fases hidrológi- 
cas del río Amazonas, permitiendo que se lleve a cabo su alimentación y reproducción (Rodríguez, 1991; Salinas, 1994).

\section{Aproximación a la migración potamodroma}

Anteriormente se pensaba que la migración en peces dulceacuícolas era relativamente extraña, ya que la mayoría de las especies se consideraban en gran parte sedentarias y mostraban movimientos locales limitados (Lucas y Baras, 2001). Aunque se conoce que las migraciones están basadas ecológicamente en su relación directa con los límites de aguas continentales o salobres, la migración potamodroma ocurre enteramente dentro de ecosistemas acuáticos continentales, abarcando hábitats lacustres como fluviales (Binder et al., 2011; Zapata y Usma, 2013; Obande et al., 2014). Excepciones notables a esta visión histórica son los grandes bagres migradores, como por ejemplo B. rousseauxii y B. vaiIlantii, las más importantes especies comerciales y las más estudiadas si de migraciones se refiere, caracterizados por su dependencia del estuario del río Amazonas como su vivero y ríos interiores como áreas de alimentación y desove (Barthem et al., 2017).

Evidencias significativas han mostrado que los peces de casi todos los hábitats dulceacuícolas y de todos los nichos ecológicos hacen movimientos migratorios en un rango de escalas espaciales y temporales. La capacidad mejorada para recopilar datos detallados sobre los movimientos de los peces también ha llevado a un cambio de los estudios descriptivos a investigaciones más basadas en hipótesis dirigidas a abordar algunas de las causas y consecuencias subyacentes de la migración (Olsson et al., 2006; Brodersen et al., 2008; Chapman et al., 2011; Skov et al., 2011). Generalmente, las especies enmarcadas en la migración potamodroma requieren de desplazamientos direccionados durante su fase reproductiva, incluyendo desoves estrictamente estacionales y relacionados con el pulso de inundación en el río (Petrere, 1985; Lowe-McConnell, 1987; Carolsfeld et al., 2004). De la misma manera, la influencia del aumento del caudal del agua juega un papel fundamental, pues los factores físicos y químicos inciden sobre la maduración y culminación del proceso reproductivo (Vazzoler, 1996; Jiménez-Segura et al., 2010).

\section{Movimientos migratorios en la Amazonia}

Desde hace algunas décadas, las investigaciones se han centrado en migraciones de larga distancia, no porque las migraciones a corta distancia sean ecológicamente o evolutivamente menos importantes, sino porque las migraciones en peces que recorren largas distancias imponen típicamente mayores desafíos comportamentales y fisiológicos que a los peces que realizan migraciones cortas (Binder et al., 2011; Duponchelle et al., 2016). Por tal motivo, el orden de los Siluriformes y su familia Pimelodidae ha tenido una implicación relevante en aspectos ecológicos y pesqueros, pues adicionalmente de que se encuentran en la mayoría de las cuencas de América del Sur, también han correspondido al segundo orden con mayor abundancia en especies para el Neotrópico, presentando mayores adaptaciones anatómicas y morfológicas (Salinas y Agudelo, 2000; Carolsfeld et al., 2004; Lasso et al., 2004, 2011).

De acuerdo a lo anterior, Lowe-McConnell (1987) logra recopilar información de acuerdo a la clasificación de los movimientos migratorios en los peces amazónicos, que están directamente relacionados con la variación de elevación y descenso del nivel de las aguas en los ríos: (Goulding, 1979; 1980; 1981; Ribeiro, 1985; Junk, 1984; Cox-Fernandes, 1988):

Migraciones motivadas por el cambio del nivel de las aguas: Estas migraciones son generalmente movimientos cortos en un espacio de centenas de metros a pocos kilómetros, siendo realizadas por las especies que colonizan las planicies de inundación. Son ocasionadas por los cambios del nivel de las aguas que permiten y facilitan el acceso a lugares de alimentación y de protección en el periodo de inundación y la salida de estos lugares durante el descenso, por lo general, están relacionadas con que han sido consideradas sedentarias.

Migraciones motivadas por fines reproductivos: Estas migraciones son realizadas por muchos Siluriformes y Characiformes, y ocurren generalmente en el inicio de la inundación. En algunos casos, las migraciones para desove se presentan solamente entre las planicies de inundación y los ríos, pero pueden ser realizadas río arriba hasta las cabeceras o más comúnmente, río abajo, siendo éste último caso, en el sentido de los ríos de aguas claras y negras para los de aguas blancas.

Migraciones motivadas por fines tróficos: Prácticamente todas las especies que realizan migraciones reproductivas también efectúan migraciones tróficas, en general, ellas son direccionadas río arriba, tal vez para compensar el desplazamiento de los ovocitos, de las larvas y de los juveniles, ocasionadas por el movimiento río abajo.

\section{Ciclo hidrológico y pulso de inundación}

El régimen de hidrológico durante el flujo de lluvias y posteriormente en el periodo de verano sobre la 
cuenca hidrográfica, se ha constituido como el factor ambiental de mayor importancia en la estructuración de las comunidades y en el funcionamiento de los sistemas de ríos y planicies de inundación (Lowe-McConnell, 1987; Junk et al., 1989) reflejándose sobre la integridad biótica y dinámica de los ensamblajes (Thomaz et al., 1997; Agostinho et al., 2001).

El ciclo hidrológico puede ser resumido en cuatro fases: aguas subiendo (inundación) aguas altas (punto máximo del caudal de agua), aguas bajando (descenso) y aguas bajas (sequía) (Lowe-McConnell, 1987). El pulso de inundación es el factor principal y responsable por la existencia de la productividad e interacción de la biota que vive en áreas periódicamente inundadas (Junk et al., 1989; Camargo y Esteves, 1996). Las precipitaciones intensas pueden inundar inmediatamente los ríos y arroyos de las cabeceras, pero los grandes ríos aguas abajo son menos influenciados por las lluvias locales y más por la precipitación total en las regiones aguas arriba que descienden en forma de una ola de inundación, ocasionando el desbordamiento de los ríos (Welcomme, 1985).

Esto podría ser un factor determinante en los cambios físicos y químicos del ambiente y como consecuencia las especies responden con una serie de adaptaciones morfológicas y fisiológicas (Junk et al., 1997) por ejemplo, en la incidencia reproductiva de individuos con diferentes patrones de desplazamiento, así lo describe Agostinho (2004) las especies de carácter sedentario con cuidado parental o fecundación interna parecen reproducirse independientemente del régimen de lluvias, salvo que las migradoras de corta distancia muestran tendencias intermedias. Pese a esto, hay una gran diferencia si se compara con los grandes migradores, puesto que presentan un desove total y tendencias a tener un elevado flujo genético como se ha estudiado en B. rousseauxii y B. vaillantii (Barthem et al., 1997; Batista, 2001) además, los peces evitan pérdidas de energía para realizar el desove en las regiones altas de la cuenca y así utilizar áreas inundables en las fases iniciales del desarrollo (Nakatani et al., 1997; Baumgartner et al., 2004; Agostinho et al., 2004).

La inundación es caracterizada por el aumento del nivel del río e igualmente por la acentuada expansión de los ambientes acuáticos en la planicie inundable, aunque la disponibilidad y la cantidad de nutrientes en zonas inundables están determinadas por el mismo ciclo (Goulding, 1980; Lowe-McConnell, 1987; Biodamaz, 2001). La importancia del pulso de inundación radica en consideración a su relación directa como uno de los factores principales que condicionan la biología y ecología de los ecosistemas amazónicos, ya que existe una dinámica en el intercambio de nutrientes y energía entre el componente acuático y terrestre (Junk et al., 1989). Esto sucede cuando el agua desborda el canal principal de los ríos hacia las zonas adyacentes y es en el inicio de este periodo que se presenta el desove de varias especies migratorias, para dispersar sus ovocitos en áreas recién inundadas (Lowe-McConnell, 1987; Montreuil et al., 2001) (Fig.1).

Luego de que la inundación cobije áreas específicas, como la vegetación baja y selva arbórea, los peces encuentran un ambiente ideal, bien sea por refugio y disponibilidad de alimento, algas planctónicas y perifíticas, que crecen en los ambientes lacustres y áreas inundadas menos sombreadas, así como también materia orgánica en descomposición, producto de algunas macrófitas acuáticas y restos de animales (Goulding, 1980; Lowe-McConnell, 1987; Goulding et al., 1988; Araújo-Lima et al., 1995; Junk et al., 1997). Durante este tiempo, los peces se encuentran muy dispersos, alimentándose intensamente y se caracteriza por ser el periodo de mayor tasa de crecimiento para las especies asociadas a las áreas inundables (LoweMcConnel 1987; Junk, 1985; Oliveira, 1996; Fabré y Saint-Paul, 1998; Vieira, 1999) (Fig.1).

Durante el pico de aguas altas, el caudal del agua alcanza su punto máximo, es caracterizado por su corta duración y por un dominio total del ambiente acuático en las planicies inundables (Ribeiro y Petrere, 1990; Barthem y Fabré, 2004). En el descenso, los peces dan inicio a una acción de agrupamiento, las especies migradoras empiezan a formar cardúmenes y a iniciar su periodo de migración y dispersión (Fig.1) que durará todo el periodo de aguas bajas hasta el inicio de la inundación (Ribeiro y Petrere, 1990) no obstante, estas especies al dirigirse al canal principal del río o para los lagos, reducen su actividad alimentar, esta etapa es denominada como "invierno fisiológico" (LoweMcConnel, 1987).

El periodo de aguas bajas es caracterizado por tener cierto grado de complejidad ecológica para la mayoría de las especies, pues el ambiente acuático es muy reducido, conllevando a una disminución del alimento y refugio (Fig.1). Estas áreas, que en algún momento estuvieron inundadas, forman un ambiente poco habitable e hipoxémico, como respuesta a la intensa descomposición de materia orgánica que consume el oxígeno disuelto en el agua y va liberando el gas sulfídrico, provocando una mortalidad de los peces que llegan a estos lugares (Santos, 1979; Lowe-McConnel, 1987). Estas variaciones en el ambiente, alteran la composición de las comunidades de peces en sistemas de tipo lacustre y de áreas de inundación, pero 
al mismo tiempo genera resiliencia en algunas especies para afrontar disturbios ocasionados por el ciclo hidrológico (Junk et al., 1989). Según Putman (1996) la resiliencia hace parte de un componente ecológico que ofrece una respuesta sobre cómo un disturbio puede modificar el comportamiento de organismos en su respectivo ambiente. Investigaciones realizadas por Bayley y Osborne (1993) y Humphries y Baldwin (2003) indican que ante condiciones adversas, las poblaciones de organismos aparentemente tienden a recuperarse y se reestablecen con rapidez después de ciertos acontecimientos naturales.

La fase de aguas bajas es un periodo mucho más favorable para los depredadores, que van en busca de cardúmenes de Characiformes y otros Siluriformes de menor talla, que en ese preciso instante estarán migrando en el canal del río o confinados en los lagos (Lowe-McConnel, 1987; Barthem y Goulding, 1997). A pesar de lo anterior, los peces desarrollaron durante el proceso evolutivo adaptaciones comportamentales, las cuales se modifican acuerdo a las condiciones del ambiente (Val y Almeida-Val, 1995). Con relación a las actividades de predación, que se presentan en aguas bajas por parte de los grandes bagres, Reid (1986) observó que los juveniles del cucharo (Sorubim lima) un pimelódidos de tamaño medio posee una coloración críptica, característica que permite que la coloración del pez se confunda con la del medio que lo rodea, haciendo que sea menos vulnerable en las planicies de inundación, es posible que sea una adaptación necesaria para los pimelódidos que usan las planicies de inundación y el canal principal del río en el inicio de sus vidas (Lowe-McConnel, 1987).

\section{Clasificación de bagres migratorios}

Las características del ambiente y el comportamiento de los peces, permite diferenciar dos grupos de especies, migradores y grandes migradores (Tabla 1) los migradores que hacen uso de los ambientes lacustres y fluviales, y los grandes migradores, relacionadas principalmente con el canal de los ríos y en algunos casos el uso de estuarios (Barthem et al., 1997; Batista, 2000).

\section{Acciones antrópicas}

La elevada capturabilidad en determinadas fases migratorias, explican el gran interés de la pesca comercial sobre las poblaciones que presentan comportamientos migratorios, no obstante, las actividades antrópicas

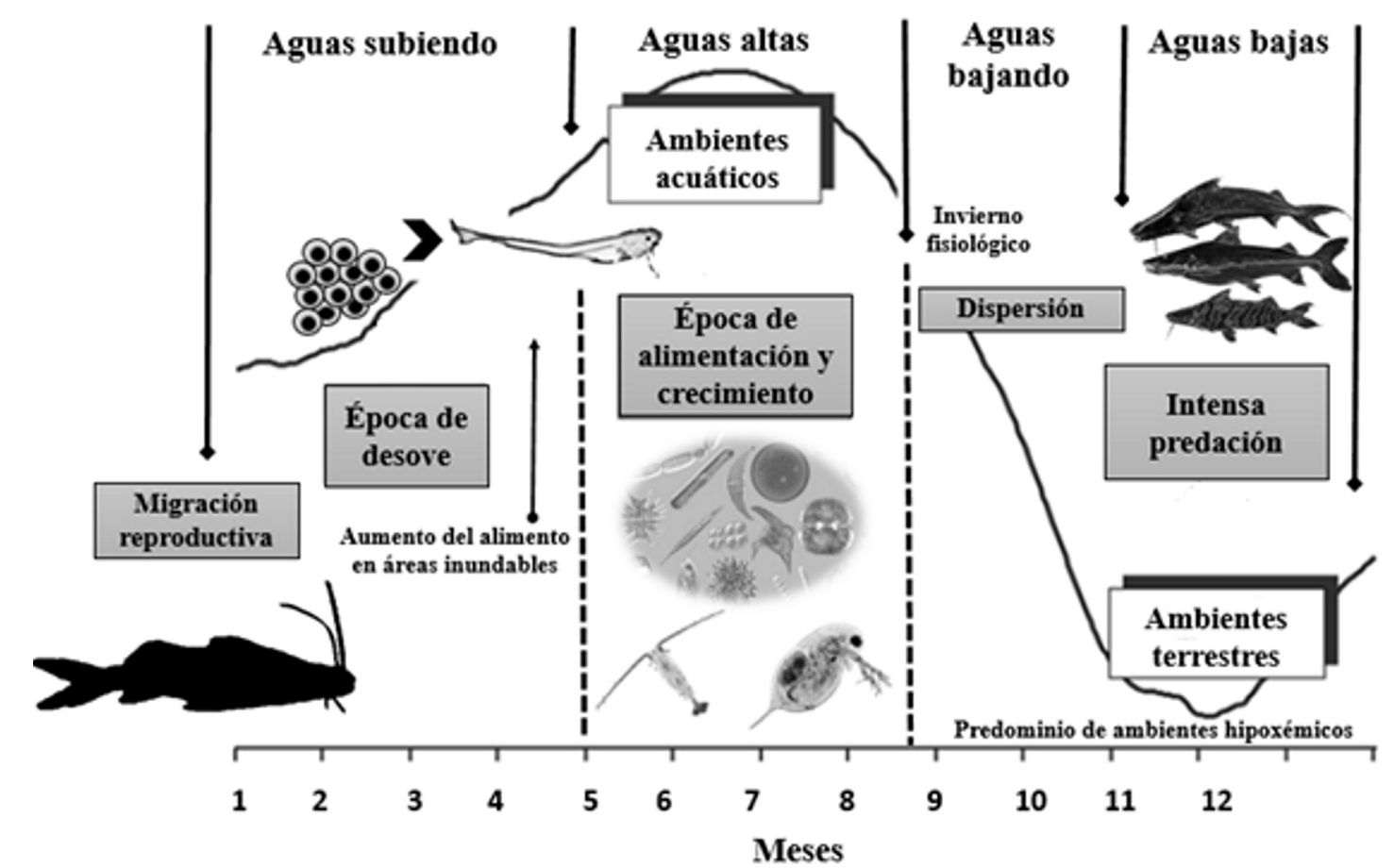

Figura 1. Dinámica del ciclo hidrológico sobre la migración en bagres (Adaptado de Lowe-McConnel, 1987; Barthem y Fabré 2003). 


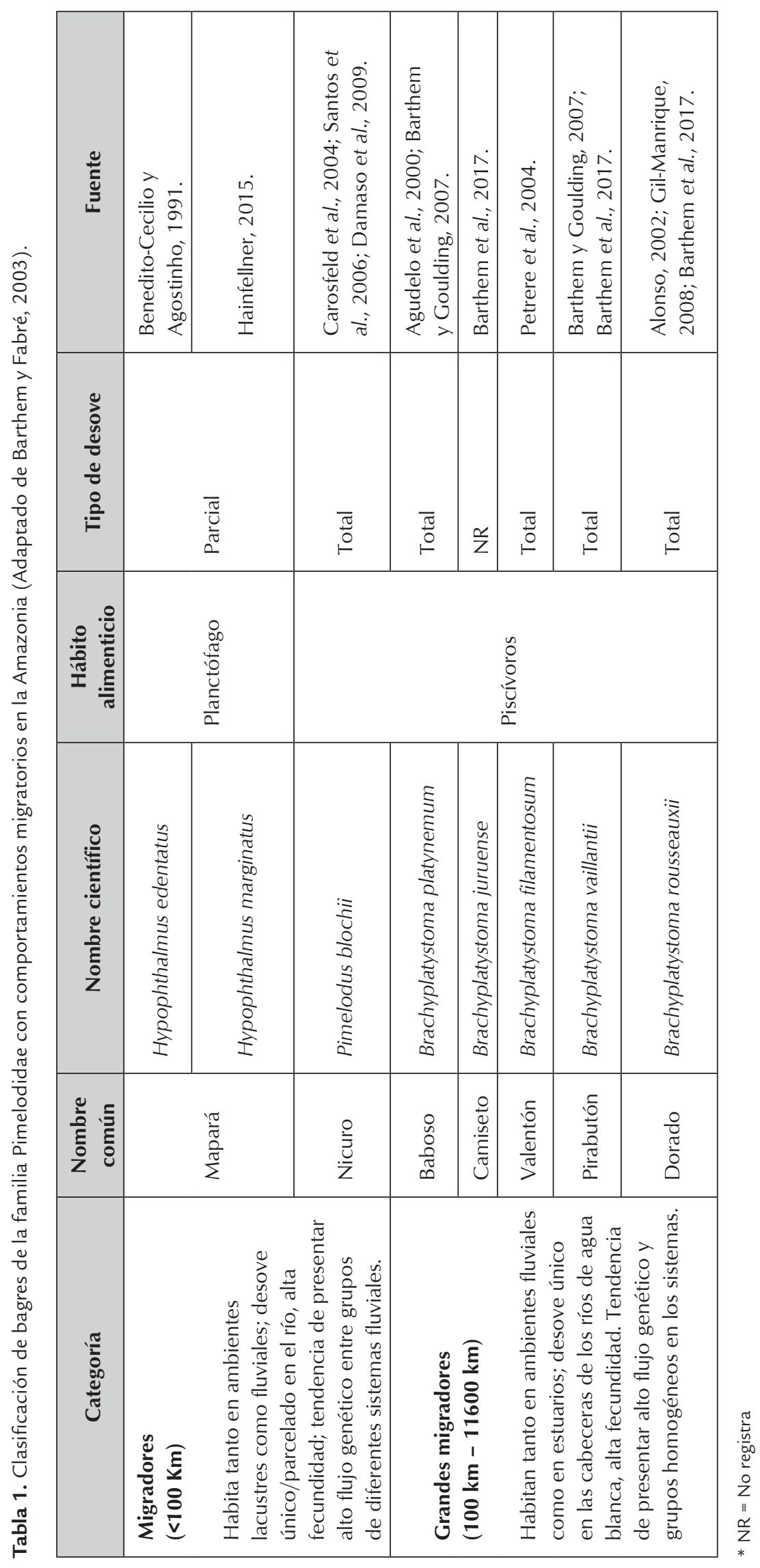


mediante la construcción de barreras que irrumpen las rutas migratorias de los peces, pueden perjudicar la abundancia del stock explorado, la geomorfología fluvial que a su vez puede alterar la erosión, el transporte y la deposición de sedimentos (Carvalho y Merona, 1986; Schilt, 2007).

La construcción de represas, además de modificar las características físicas y químicas del agua y controlar su flujo (Agostinho et al., 2007) impide el libre tránsito de los peces por el río, haciendo que muchas especies no puedan efectuar su reproducción, constituyéndose como uno de los mayores peligros para la ictiofauna, eliminando bien sea especies diádromas y potamodromas de diferentes cuencas fluviales (Agostinho et al., 2005; Williams et al., 2012). De tal manera que, las estrategias desarrolladas por los peces muchas veces son afectadas por alteraciones en el ambiente, tales como las barreras que transforman ambientes lóticos en lénticos (Giamas et al., 2004) obligando a que las comunidades bióticas de su área de influencia deban adaptarse a nuevas condiciones para no ser eliminadas del sistema (Braga, 2001) además, como lo afirman Fearnside et al., (2014) las modificaciones en el hábitat principalmente por medio de represamientos, disminuye la variabilidad genética de las especies, reduciendo las tasas de crecimiento poblacional y aumentando en gran medida el riesgo de extinción.

Con el objetivo de mitigar el impacto negativo de las barreras sobre la migración de los peces, fueron construidas en Brasil, a partir del inicio del siglo XX, innumerables escaleras de peces, a fin de posibilitar la libre migración, río arriba y en la época reproductiva, pero a pesar de esto, su implementación ha sido realizada sin criterios técnicos, trayendo como consecuencia riesgos directos para la conservación de la ictiofauna local (Silva et al., 2012). Según Santos et al., (2007) el sistema de transposición de peces es de tipo escalera con escalón, este sistema parece ser mucho más simple si es comparado con otros modelos. Se constituye en una serie de depósitos o tanques escalonados secuencialmente, en donde los depósitos se separan por escalones. En la migración, los peces poco a poco van atravesando los escalones, pasando entre los depósitos o tanques durante la piracema, nadando por la lámina de agua bajando o saltando (Santos et al., 2007). Los escalones tienen la finalidad de controlar la permanencia de niveles de agua y esparcir la energía, haciendo que ésta sea disipada para favorecer la ascensión de los peces sin causar su cansancio (Makrakis et al., 2011) pero se requiere que las técnicas empleadas incluyan monitorización de la recolección cualitativa de informaciones biológicas que indiquen cuantos y de qué forma los peces transponen el obstáculo (Pompeu et al., 2012).

\section{Conclusión}

La cuenca Amazónica ha sido una de los sistemas más preservados del mundo y es caracterizado por presentar alto endemismo y diversidad de especies de peces con diferentes aspectos reproductivos y comportamentales, pero infortunadamente, los elevados costos de este tipo de investigaciones implican desconocimiento de sus rutas migratorias. Igualmente, estudios recientes han indicado que las pesquerías podrían estar disminuyendo debido a la sobrepesca y que las barreras impuestas por la acción antrópica en los cursos de los ríos aguas arriba amenazan constantemente las migraciones reproductivas. Por consiguiente, en la última década, una serie de reportes genético-geográficos y microquímicos han surgido para dar respuesta a estos sucesos migratorios y de alta dispersión a lo largo del ciclo de vida de poblaciones de bagres pimelódidos en los ecosistemas acuáticos de la cuenca amazónica. Entre ellos, se puede mencionar lo realizado por Telles et al., (2014), quienes a través de análisis espaciales para investigar la variación genética molecular (utilizando 7 marcadores de microsatélites) de Pseudoplatystoma punctifer, hicieron uso de muestras obtenidas de 15 localidades a lo largo del río Madeira y Solimões, obteniendo como resultado una disminución en la similitud genética, la cual varía entre la distancia de las poblaciones, así, se concluyó, que el patrón revelado tiene implicaciones importantes para establecer estrategias para mantener la diversidad genética en la especie, especialmente teniendo en cuenta las amenazas por los impactos causados por la construcción de grandes presas en este sistema fluvial. Luego Hegg et al., (2015), mediante microquímica de otolitos, describen los comportamientos migratorios de las tres especies de bagres migratorias más populares y comercialmente importantes, dorado (B. rousseauxii), pirabutón (B. vaillantii) y valentón (B. filamentosum). Las colectas se realizaron en la desembocadura del río Amazonas y la Amazonia central y se usaron firmas de isótopos de estroncio $(87 \mathrm{Sr} / 86 \mathrm{Sr}$ ) registradas en los otolitos para determinar la ubicación de la cría temprana y posterior. Los resultados indicaron que los juveniles exhiben diversas estrategias de crianza, tanto los que se desarrollan en ambientes río arriba y en el estuario. Esto contrasta con el entendimiento prevaleciente de que los juveniles se crían en el estuario antes de migrar río arriba, sin embargo, cuenta con el respaldo de algunos datos de pesquerías que han indicado la presencia de historias de vida de desove y cría alternativos. Todo lo anterior, conduce a formular investigaciones encami- 
nadas a fortalecer el conocimiento sobre comportamiento migratorio de las especies, ya que a partir de ellas se podrían identificar zonas que no han sido exploradas y que funcionan como áreas de llegada para el desove y alimentación de especies con importancia comercial para las pesquerías, permitiendo identificar aún más sus estrategias reproductivas.

\section{Referencias}

Agostinho AA, Gomes LC, Zalewski M. The importance of floodplains for the dynamics of fish communities of the upper River Paraná. Ecohydrol Hydrobiol. 2001;1:209-217.

Agostinho AA, Gomes LC, Suzuki HI, Júlio Jr HF. 2003. Migratory fish from the upper Parana river basin, Brazil. In: Carolsfeld J.; Harvey B.; Ross C. \& Baer A. (eds). Migratory fishes of South America: biology, social importance and conservation status. Victoria, World Fisheries Trust, The World Bank and The International Development Research Centre. p. 19-98.

Agostinho AA, Gomes LC, Veríssimo S, Okada EK. Flood regime and fish: effects on spawning, recruitment and attributes of the assemblages in the upper Paraná River floodplain. RRev Fish Biol Fish. 2004;14:11-19.

Agostinho AA, Marques EE, Agostinho CS, Almeida DA, Oliveira RJ, Melo JRB. Fish ladder of Lajeado Dam: migrations on one-way routes? Neotrop Ichthyol. 2007;5(2):121-130.

Agudelo E, Salinas Y, Sánchez CL, Muñoz - Sosa DL, Alonso JC, Arteaga ME, Rodríguez OJ, Anzola NR, Acosta LE, Núñez M, Valdés H. 2000. Bagres de la Amazonia colombiana: un recurso sin fronteras. Fabre, N. N., Donato, J. C. y J. C. Alonso (Eds.). Instituto Amazónico de Investigaciones Científicas SINCHI. Programa de Ecosistemas Acuáticos. Bogotá., p. 252.

Agudelo E, Sánchez CL, Acosta LE, Mazorra A, Alonso JC, Moya LA, Mori LA. 2006. La pesca y la acuicultura en la frontera colomboperuana del río Putumayo. Pp 79-98. In: Agudelo, E., Alonso, J.C \& L.A. Moya (Eds). 2006. Perspectivas para el ordenamiento de la pesca y la acuicultura en el área de integración fronteriza colombo-peruana. Instituto Amazónico de Investigaciones Científicas Sinchi - Instituto Nacional de Desarrollo del Perú. Bogotá, D.C.

Agudelo E, Alonso JC, Sánchez L. 2009. La utilización de los recursos ícticos en la Amazonia Sur de Colombia: una estrategia de vida, de ocupación y renta. Pp. 237-247. In: Bernal, H., C. Sierra \& M. Angulo (Eds.). Amazonia y Agua: Desarrollo sostenible en el siglo XXI, UNESCO. Servicio Editorial de la Unesco Etxea, Bilbao, España.

Agudelo E. 2015. Bases científicas para contribuir a la gestión de la pesquería comercial de bagres (Familia Pimelodidae) en la Amazonia Colombiana y sus zonas de frontera. Tesis Doctoral Institut de Ciència I Tecnologia Ambientals - ICTA, Universitat Autònoma de Barcelona.

Allan JD, Abell R, Hogan Z, Revenga C, Taylor BW, Welcomme RL, Winemiller K. Overfishing of Inland Waters. BioScience, 2005;55(12):1041-1051.

Almeida-Val VMF, Val AL, Walker I. 1999. Long- and short-term adaptation of Amazon fishes to varying O2-levels: intra-specific phenotypic plasticity and interspecific variation. In Biology of Tropical Fishes (Val, A. L. \& Almeida-Val, V. M. F., eds), pp. 185-206. Manaus: INPA: 185-206.

Almeida OT. 2006. A Indústria pesqueira na Amazônia. Manaus. Ibama/Provarzea.

Alonso JC. 2002. Padrão espaço - temporal da estrutura populacional e estado atual da exploração pesqueira da dourada Brachyplatystoma rousseauxii, Castelnau, 1855 (Siluriformes: Pimelodidae) no sistema Estuário-Amazonas-Solimões. Tese de Doutorado. INPA/UFAM, Manaus, p. 217.

Araújo-Lima CARM, Agostinho AA, Fabré NN. 1995. Trophic aspects of fish communities in Brazilian River and Reservoirs. Tundisi, J.G., Bicudo, C.E.M. and Matsumura-Tundisi, T. Limnology in Brazil. 105-136.

Barthem R, Guerra H, Valderrama M. 1994. Diagnóstico de los recursos hidrobiológicos de la Amazonia TCA Secretaria Pro Tempore, p. 162.

Barthem RB, Goulding M. 1997. The Catfish Connection: Ecology, Migration, and Conservation of Amazon Predators (Biology and Resource Management in the Tropics Series.) Columbia Univ Press, p. 184.

Barthem RB, Petrere Jr M, Isaac VJ, Ribeiro MCLB, McGrath D, Vieira I, Valderrama M. 1997. A pesca na Amazônia: problemas e perspectivas para o seu manejo. In: Valladares-Padua, C.; Bodmer, R. \& Cullen Jr., L. (orgs.). Manejo e Conservação da Vida Silvestre no Brasil. MCT-CNPq, Sociedade Civil Mamirauá, Rio de Janeiro, p. 173-184.

Barthem RB, Fabré NN. 2004. Biologia e diversidade dos recursos pesqueiros da Amazônia. In: Ruffino, M.L. (coord.). A pesca e os recursos pesqueiros na Amazônia brasileira. Ibama/Provárzea. p. 17-62.

Barthem RB, Goulding M. 2007. An unexpected ecosystem: the Amazon revealed by the fisheries. Gráfica Biblos, Lima/Botanical Garden Press.

Barthem R. 2013. Estado natural, amenazas y tendencias de los recursos pesqueros en la Amazonia Brasileña. In: Hacia el manejo de las pesquerías en la cuenca amazónica, perspectivas transfronterizas. Collado L, Castro E, Hidalgo M (Eds.) p. 13-17.

Barthem RB, Goulding M, Leite RG, Cañas C, Forsberg B, Venticinque E, Petry P, Ribeiro LDB, Chuctaya J, Mercado A. Goliath catfish spawning in the far western Amazon confirmed by the distribution of mature adults, drifting larvae and migrating juveniles. Scientific Reports, 2017;7:41784.

Batista J da S. 2001. Estimativa da variabilidade genética intra-específica da dourada - Brachyplatystoma rousseauxii Castelnau 1855 (Pimelodidae - Siluriformes) no sistema Estuário-Amazonas-Solimões. Dissertação de Mestrado, INPA, Manaus, p. 97.

Batista VS, Petrere M Jr. Characterization of the comercial fish production landed at Manaus, Amazonas state, Brazil. Acta Amaz., 2003;33:53-66.

Batista J, Formiga-Aquino K, Pires-Farias I, Alves-Gomes J. 2005. Variabilidade genética da Dourada e da Piramutaba na bacia Amazônica. In: O manejo da pesca dos grandes bagres migra- 
dores Piramutaba e Dourada no Eixo Solimões-Amazonas. Fabré NN, Barthem R (Org). Manaus: Ibama/ProVárzea.

Baumgartner G, Nakatani K, Gomes LC, Bialetzki A, Sanches PV, Makrakis MC. Identification of spawning sites and natural nurseries of fishes in the upper Paraná River, Brazil. Environ Biol Fishes. 2004; 71:115-125.

Bayley PB, Petrere Jr M. 1989. Amazon fisheries: Assessment methods, current status, and management options. Can Spec Publ Fish Aquat Sci, 106: 385-398.

Bayley PB, Osborne LL. 1993. Natural rehabilitation of streams fish populations in an Illinois catchment. Freshw Biol. 1993;29:295300.

Benedito-Cecilio E, Agostinho AA. 1991. Biologia reprodutiva de Hypophthalmus edentatus (Spix, 1829) (Osteichthyes, Siluriformes) no reservatório de Itaipu - PR. II. Estrutura dos ovários e escala de maturidae. Revista UNIMAR, 212-217.

Binder TR, Cooke SJ, Hinch SG. 2011. The Biology of Fish Migration. In: Farrell AP (Ed.), Encyclopedia of Fish Physiology: From Genome to Environment, volume 3. San Diego: Academic Press, p. 1921-1927.

BIODAMAZ. 2001. Estrategia Regional de la Diversidad Biológica Amazónica. Documento Técnico № 01 - Serie Biodamaz - IIAP, Iquitos, Perú.

Braga FMS. Reprodução de peixes (Osteichthyes) em afluentes do reservatório de Volta Grande, Rio Grande, sudeste do Brasil. Iheringia, 2001;91(1):67-74.

Brodersen J, Nilsson PA, Hansson LA, Skov C, Bronmark C. Condition dependent individual decision-making determines cyprinid partial migration. Ecology. 2008; 89:1195-1200.

Brum SM, da Silva VMF, Rossoni F, Castello L. Use of dolphins and caimans as bait for Calophysus macropterus (Lichtenstein, 1819) (Siluriforme: Pimelodidae) in the Amazon. Journal of Applied Ichthyology, 2015;31(4):675-680.

Camargo AFM, Esteves FA. Influence of water level variation on biomass and chemical composition of aquatic macrophyte Eichhornia azurea (Kunth) in an oxbow lake of the rio Mogi-Guaçu (São Paulo, Brasil). Arch Hydrobiol. 1996;135:423-432.

Carolsfeld J, Harvey B, Baer A, Ross C. 2004. Migratory fishes of South America: Biology, social importance and conservation status. World Fisheries Trust/Banco Mundial/IDRC. Victoria, Canada.

Carvalho JL, Merona B. Estudos sobre dois peixes migratórios do baixo Tocantins, antes do fechamento da barragem de Tucuruí. Amazoniana, Kiel. 1986;9(4):595-607.

Castello L, McGrath DG, Hess LL, Coel MT, Lefebvre1 PA, Petry P, Macedo MN, Reno VF, Arantes CC. 2013. The vulnerability of Amazon freshwater ecosystems. Conservation Letters, p. 1-13.

Chapman BB, Hulthen K, Blomqvist DR, Hansson LA, Nilsson JA, Brodersen J, Nilsson PA, Skov C, Bronmark C. To boldly go: individual differences in boldness influence migratory tendency. Ecology Letters. 2011;14:871-876.
Cooke SJ, Paukert C, Hogan Z. Endangered river fish: factors hindering conservation and restoration. Endangered Species Res. 2012; 17: 179-191.

Cox-Fernandes C. 1988. Estudos de migrações laterais de peixes no sistema lago de Rei (ihla do Carneiro) - AM, BR. Dissertação de Mestrado. INPA/FUA, Manaus, p. 170.

Cox-Fernandes C, Podos J, Lundberg JG. Amazonian ecology: tributaries enhance the diversity of electric fishes. Science. 2004;305:1960-1962.

Damaso JA, Ipuchima, Santos A. 2009. Conocimiento local indígena sobre los peces de la Amazonia: Lagos de Yahuarcaca. Santiago Duque (Ed.). Universidad Nacional de Colombia. Sede Amazonia. Bogotá. 68 p.

De la Hoz-M J, Manjarrés-Martínez L. 2016. Estadísticas de desembarco y esfuerzo de las pesquerías artesanales e industriales de Colombia en los sitios y puertos pesqueros monitoreados por el SEPEC durante el período julio a diciembre de 2016. Autoridad Nacional de Acuicultura y Pesca (AUNAP), Santa Marta, p. 60.

Diegues ACS. 2004. A Pesca Constituindo Sociedades. São Paulo: Núcleo de Apoio à Pesquisa Sobre Populações Humanas e Áreas Úmidas Brasileiras/USP.

Dingle H, Drake VA. What is Migration?. Bioscience. 2007;57:113121.

Duponchelle F, Pouilly M, Pécheyran C, Hauser M, Renno JF, Panfili J, Darnaude AM, García-Vasquez A, Carvajal-Vallejos F, García-Dávila C., Doria C, Bérail S, Donard A, Sondag F, Santos RV, Nuñez J, Point D, Labonne M, Baras E. Trans-Amazonian natal homing in giant catfish. J Appl Ecol. 2016;53(5):15111520.

Fabré NN, Alonso JC. Recursos ícticos no Alto Amazonas: sua importancia nas populações riberinhas. Boletin do Museu Paraense Emilio Goeldi. Sér Zool. 1998;1:19-55.

Fabré NN, Saint Paul U. Annulus formation on scales and seasonal growth of the Central amazonian anostomid Schizodon fasciatus. J Fish Biol. 1998;53:1-11.

Fearnside PM. Impacts of Brazil's Madeira River dams: unlearned lessons for hydroelectric development in Amazonia. Environ Sci Policy. 2014;38:164-172.

Galvis G, Mojica Jl, Camargo M. 1997. Peces del Catatumbo. Ecopetrol: OXY: SHELL- Asociación Cravo Norte. D, Vinni Edi. Ltda., Santa Fe de Bogotá, p. 118.

Giamas MTD, Campos EC, Câmara JJC, Vermulm Jr H, Barbieri G. A ictiofauna da represa de Ponte Nova, Salesópolis (São Paulo) bacia do alto Tietê. Bol Inst Pesca. 2004;30:25-34.

Gil-Manrique BD. 2008. Aspectos de la biología reproductiva del Dorado Brachyplatystoma rousseauxii Castelnau, 1855 (PISCES: PIMELODIDAE), en el área fronteriza Brasil - Colombia - Perú del río Amazonas como instrumento de manejo y conservación. Trabajo de grado, Programa de Biología Marina, Universidad de Bogotá Jorge Tadeo Lozano. Bogotá, Colombia, p. 138.

Goulding M. 1979. Ecologia da pesca do Rio Madeira. CNPq/INPA, Belém, p. 172 
Goulding M. 1980. The Fishes and the Forest: Explorations in Amazonian Natural History. Los Angeles: University of California.

Goulding M. 1981. Man and Fisheries on An Amazon Frontier. Developments in Hidrobiology, no. 4. The Hague: Dr W. Junk.

Goulding M, Carvalho ML, Ferreira EJG. 1988. Rio Negro: Rich Life in Poor Water: Amazonian Diversity and foodchain Ecology As Seen Through Fish Communities. The Hague: SPB Academic Publishing, p. 200.

Granado-Lorencio C, Lima CRMA, Lobón-Cerviá J. Abundance-distribution relationships in fish assembly of the Amazonas floodplain lakes. Ecography. 2005;28:515-520.

Gualdrón Silva MI. 2002. Plan de manejo de los recursos ictiológicos y pesqueros en el rio grande de la Magdalena y sus zonas de amortiguación. Ajuste del Documento Recursos Hidrológicos, Ictiológicos y Pesqueros en la Cuenca Magdalena- Cauca Diagnostico (caracterización) y Estrategias de Política para la formulación del POMIM. Bogotá: Autoridad Nacional de Acuicultura y Pesca (Aunap).

Hainfellner P. 2015. Ciclo reprodutivo e indução hormonal de fêmeas de mapará (Hypophthalmus marginatus) no baixo Rio Tocantins, à jusante da barragem de Tucuruí. Tese (Doutorado) apresentada ao Programa de Pós-graduação em Aquicultura do Centro de Aquicultura da UNESP-CAUNESP, p. 103.

Hegg JC, Giarrizzo T, Kennedy BP. 2015. Diverse early life-history strategies in migratory Amazonian catfish: implications for conservation and management. PLoS One, 10, e0129697.

Humphries P, Baldwin DS. Drought and aquatic ecosystems: an introduction. Freshwater Biol. 2003;48:1141-1146.

Iriarte V, Marmontel M. Insights on the use of dolphins (boto, Inia geoffrensis and tucuxi, Sotalia fluviatilis) for bait in the piracatinga (Calophysus macropterus) fishery in the Western Brazilian Amazon. J Cetacean Res Manage. 2013;13(2):163-173.

Jiménez-Segura LF, Palacio J, Leite R. River flooding and reproduction of migratory fish species in the Magdalena River basin, Colombia. Ecol Freshw Fish. 2010;19:178-186.

Junk WJ, Soares GM, Carvalho, FM. Distribution of fish species in a lake of the Amazon river floodplain near Manaus (lago Camaleão), with special reference to extreme oxygen conditions. Amazoniana. 1983;7:397-431.

Junk WJ. 1984. Ecology, fisheries and fish culture in Amazoniana. In: The Amazon. Limnology and Landscape Ecology of Mighty Tropical River and its Basin, ed. H, Sioli, Dordrecht: Dr W. Junk, p. 443-476.

Junk WJ. Temporary fat storage, an adaptation of some fish species to the water level fluctuations and related environmental changes of the Amazon system. Amazoniana. 1985;9:315351.

Junk WJ, Bayley, PB, Sparks RE. 1989. The flood pulse concept in river-floodplain systems. In "Proceedings of the International Large River Symposium" (Dodge, D. P., Ed.), Vol. 106. Canadian Special Publication of Fisheries and Aquatic Sciences, Canada, p. 110-127.
Junk WJ, Soares MGM, Saint-Paul U. The fish. In Junk, W.J. (ed.) The Central Amazon Floodplain: Ecology of a pulsing system. Ecol Stud. 1997; 126:385-408.

Lasso C. 2004. Los peces de la Estación Biológica El Frío y Caño Guaritíco (Estado Apure), Llanos del Orinoco, Venezuela. Publicaciones del Comité Español del Programa Hombre y BiosferaRed Ibero MAB, Unesco. Sevilla.

Lasso C, Agudelo-Córdoba E, Jiménez-Segura LF, Ramírez-Gil H, Morales-Betancourt M, Ajiaco-Martínez RE, de Paula Gutiérrez F, Usma JS, Muñoz SE, Sanabria AI (Eds.). 2011. I. Catálogo de los recursos pesqueros continentales de Colombia. Serie Editorial Recursos Hidrobiológicos y Pesqueros Continentales de Colombia. Instituto de Investigaciones de Recursos Biológicos Alexander von Humboldt. Bogotá, D.C. Colombia. P. 715.

Lowe-McConnell RH. 1987. Ecological Studies in Tropical Fish Communities. Cambridge University Press, p. 382.

Lucas MC, Baras E. 2001. Migration of freshwater fishes. Blackwell Science, Oxford.

Makrakis S, Miranda LE, Gomes LC, Makrakis MC, Junior HMF. Ascent of neotropical migratory fish in the Itaipu reservoir fish pass. River Res Appl. 2011;27:511-519.

Mehner T, Kasprzak P. Partial diel vertical migrations in pelagic fish. J Anim Ecol. 2011;80:761-770.

McDowall RM. 1988. Diadromy in Fishes. London: Cromm Hill.

Montreuil V, García A, Rodríguez R. Biología reproductiva de Prochilodus nigricans (boquichico), en la Amazonía peruana. Folia Amazónica. 2001;12(1-2):5-13.

Myers GS. Usage of anadromous, catadromous and allied terms for migratory fishes. Copeia. 1949;2:89-97.

Nahagama Y, Yoshikuni M, Yamashita M, Tokumoto T, Katsu Y. Regulation of oocyte growth and maturation in fish. Curr Top Dev Biol. 1995;30:103-145.

Nahagama Y, Yamashita M. Regulation of oocyte maturation in fish. Dev Growth Differ. 2008;50:195-219.

Nakatani K, Baumgartner G, Cavicchioli M. 1997. Ecologia de ovos e larvas de peixes. In: Vazzoler, A. E. A. De M.; Agostinho A. A. \& Hahn, N. S. (Ed.) A Planície de Inundação do alto rio Paraná: Aspectos Físicos, Biológicos e Socioeconômicos. EDUEM, Maringá, p. 281-306.

Nolan KS, Fabré N, Batista VS. Landscape variables affecting fishery yield in lake systems of the Central Amazon region, Brazil. J Appl Ichthyol. 2009;25(3): 294-298.

Obande RA, Dambo A, Adah PM. Migration in fishes: a review. Nig J Fish Aqua. 2014;2(1):1-5.

Oliveira BI. 1996. Determinação da idade e aspectos da dinâmica populacional do Curimatã Prochilodus nigrans (Pisces; Prochilodontidae) da Amazônia Central. Dissertação. INPA, p. 90.

Olsson IC, Greenberg LA, Bergman E, Wysujack K. Environmentally induced migration: the importance of food. Ecol Lett. 2006;9:645-651. 
Pazin VFV, Magnusson WE, Zuanon J, Mendonça FP. Fish assemblages in temporary ponds adjacent to "terra-firme" streams in Central Amazonia. Freshw Biol. 2006;51:1025-1037.

Petrere M. 1985. Migraciones de peces de agua dulce en América Latina: algunos comentarios, COPESCAL, FAO, Rome. Documento Ocasional, p. 1-11.

Petrere Jr M, Barthem RB, Agudelo E, Corrales, B. Review of the large catfish fisheries in the upper Amazon and the stock depletion of Piraiba (Brachyplatystoma filamentosum Lichtenstein). Rev Fish Biol Fish. 2004;14:403-414.

Petry PB, Bayley PB, Markle DF. Relationships between fish assemblages, macrophytes and environmental gradients in the Amazon River floodplain. J Fish Biol. 2003;63:547-579.

Pompeu PS, Agostinho AA, Pelicice FM. Existing and future challenges: the concept of successful fish passage in South America. River Res Appl. 2012;28:504-512.

Putman RJ. 1996. Community Ecology. Chapman \& Hall, Londres. $178 \mathrm{p}$.

Reid SB. Cryptic adaptations of small juvenile catfishes Sorubim lima (Pimelodidae) in Venezuela. Biotropica. 1986;18(1):86-88.

Ribeiro MCLB. 1983. As migracões dos jaraquis (Pisces, Prochilondontidae) no Río Negro, Amazonas, Brazil. Master`s thesis, INPA, Manaus, Brazil.

Ribeiro MCLB, Petrere Jr M. Fisheries ecology and management of the jaraqui (Semaprochilodus taeniurus, S. insignis) in central Amazônia. Regul Rivers: Res Manag. 1990;5:195-215.

Rodríguez C. 1991. Bagres, Malleros y Cuerderos en el bajo río Caquetá. Bogotá. Tropenbos - Colombia.

Rossetti DF, Toledo PM. 1998. Evolution of ecosystems in Amazonia during the last 25 million years. In Amazonia heaven of a new world. Freitas, M. L. D. (Ed.). Editora Campus, Rio de Janeiro, p. 23-32.

Ruffino ML, Barthem RB. Perspectivas para el manejo de los bagres migradores de la Amazonía. Santa Fe de Bogotá, Boletín Científico. 1996;94:19-28.

Salinas Y. 1994. Aspectos de la biología pesquera de las poblaciones de los grandes bagres en el sector colombiano del río Amazonas. Bogotá. Tesis. Universidad Nacional Pedagógica.

Salinas Y, Agudelo E. 2000. Peces de importancia económica en la cuenca Amazónica colombiana. Sinchi - Editorial Scripto, Bogotá

Santos UM. Observações limnológicas sobre a asfixia e migração de peixes na Amazônia central. Ciência e Cultura. 1979;31:1033 1040.

Santos G, Ferreira E, Zuanon J. 2006. Peixes comerciais de Manaus. IBAMA/AM; ProVarzea, p. 135.

Schilt CR. Developing fish passage and protection at hydropower dams. Appl Anim Behav Sci. 2007;104:295-325.

Silva LGM, Nogueira LB, Maia BP, Resende LB. Fish passage postconstruction issues: analysis of distribution, attraction and passage efficiency metrics at the Baguari Dam fish ladder to approach the problem. Neotrop Ichthyol. 2012;10(4):751-762.
Sioli H. 1967. Studies in Amazonian Waters. Atas do simpósio sobre a Biota Amazônica. 3. Limnologia, 9 - 50.

Sioli H. 1984. The Amazon and its main affluents: Hydrogeography, morphology of the river courses and river types. In "The Amazon. Limnology and Landscape Ecology of a Mighty Tropical River and Its Basin" (Sioli, H., Ed.). W. Junk, Dordrecht, p. 127-165.

Skov C, Baktoft H, Brodersen J, Bronmark C, Chapman BB, Hansson LA, Nilsson PA. Sizing up your enemy: individual predation vulnerability predicts migratory probability. Proc R Soc Lond, B Biol Sci. 2011;278:1414-1418.

Santos HA, Pompeu PS, Martinez CB. Swimming performance of the migratory Neotropical fish Leporinus reinhardti (Characiformes: Anostomidae). Neotrop. Ichthyol. 2007;5:139-146.

Soares MGM, Almeida RG, Junk WJ. The tropic status of the fish fauna in lago Camaleão e macrophyte dominated Floodplain Lake in the middle Amazon. Amazoniana. 1986;9(4):511-526.

Telles MPDC, Collevatti RG, Braga RDS, Castro TG, Costa MCD, Silva Júnior NJD, Barthem R, Diniz Filho JAF. Geographical genetics of Pseudoplatystoma punctifer (Castelnau, 1855) (Siluriformes, Pimelodidae) in the Amazon Basin. Genet Mol Res. 2014;13(2):3656-3666.

Thomaz SM, Roberto MC, Bini LM. 1997. Caracterização limnológica dos ambientes aquáticos e influência dos níveis fluviométricos. In: A. E. A. M. Vazzoler, A. A Agostinho, N. S. Hahn. A planície de Inundação do alto rio Paraná. EDUEM, UEM - Nupélia, p. 73-102.

Val AL, Almeida-Val VMF. 1995. The Amazon ichthyofauna. In: Val, A.L.; Almeida-Val, V.M.F. (Eds). Fishes of the Amazon and their environment: physiological and biochemical aspects. SpringerVerlang, Berlim, Heidelbeg. p. 29-30.

Val AL, Almeida-Val D, Randall DL. 2005. Tropical environment. In Val, A. L., Almeida-Val, D., \& Randall, D. L. (Eds). The physiology of tropical fishes. Pp. 1-45.

Vazzoler AEAM. 1996. Biologia da Reprodução de Peixes Teleósteos: Teoria e Práctica. São Paulo: Editora DAUFSC.

Vieira EFV. 1999. Determinação da idade e crescimento do jaraqui de escama grossa (Semaprochilodus insignis) na Amazônia Central. Dissertação de mestrado INPA/ FUA, p. 77.

Webb S. Biological implications of the middle Miocene Amazon seaway. Science. 1995;269:361-362.

Welcomme RL. 1985. River Fisheries. FAO Fisheries Technical Paper. Roma, Food and Agriculture Organization of the United Nations, 262, p. 330.

Welcomme RL, Bartley DM. Current approaches to the enhacement of fisheries. Fish Manag Ecol. 1998;5:351-382.

Williams JG, Armstrong G, Katapodis C, Larinier M, Travade F. Thinking like a fish: a key ingredient for development of effective fish passage facilities at river obstructions. River Res Appl. 2012;28:407-417.

Zapata LA, Usma JS (Editores). 2013. Guía de las especies Migratorias de la Biodiversidad en Colombia. Peces. Vol. 2. Ministerio de Ambiente y Desarrollo Sostenible / WWF-Colombia. Bogotá, D.C. Colombia. p. 486. 